\section{Progressive Encephalopathy and Central Hypoventilation Related to Homozygosity of NDUFVI Nuclear Gene, a Rare Mitochondrial Disease}

Keywords: Progressive encephalopathy; Central hypoventilation; Nuclear mitochondrial disease; NDUFV1 gene

\begin{abstract}
Background: Mitochondrial diseases are a group of disorders caused by dysfunctional organelles that generate energy for our body. Mitochondria small double-membrane organelles found in every cell of the human body except red blood cells. Mitochondrial diseases are sometimes caused by mutations in the mitochondrial DNA that affect mitochondrial function. Other mitochondrial diseases are caused by mutations in genes of the nuclear DNA, either as Autosomal recessive or Autosomal dominant inheritance pattern whose gene products are imported into the mitochondria (mitochondrial proteins) as well as acquired mitochondrial conditions. We describe a clinica presentation of a patient with an autosomal recessive mitochondrial disease due to a homozygous mutation in the nuclear gene, NDUFV 1 gene (OMIM: 618225).
\end{abstract}

Case presentation: In the present study, we report 36 months old girl from Saudi origin product of consanguineous marriage. With the clinical presentation of failure to gain normal developmental milestones, neuromotor regression, frequent attacks of the unexplained decreased level of consciousness and encephalopathy associated with central hypoventilation. There is a strong family history of similar presentation with early childhood deaths in two other siblings with no healthy kids for the couple. The girl evaluated thoroughly to reach a specific diagnosis, including clinical, radiological and biochemical work-up. However, we got an explanation for this phenotype through molecular genetic testing and couple referred for the Preimplantation Genetic Diagnosis.

Result: The constellation of clinical presentation and radiological finding confirmed by a Molecular test showed a homozygous missense mutation c. $1268 \mathrm{C}>\mathrm{T}$ p. (Thr423Met) in the NDUFV 1 gene (OMIM: $618225)$ which is consistent with autosomal recessive mitochondrial disease.

\section{Introduction}

Mitochondrial diseases are a group of disorders caused by dysfunctional mitochondria, the organelles that generate energy for our body cells. Mitochondria are small double membrane organelles found in every cell of the human body except red blood cells. Mitochondrial diseases are can be caused by mutations in the mitochondrial DNA that affect mitochondrial function. Other mitochondrial diseases are caused by mutations in genes of the nuclear DNA, either as Autosomal recessive or Autosomal dominant inheritance pattern whose gene products are imported into the mitochondria (mitochondrial proteins) as well as acquired mitochondrial conditions due to adverse effects of drugs, infections, or other environmental causes [1]. Mitochondrial disease is one

\section{Journal of}

Pediatrics \& Child Care

\author{
AL-Buali MJ*, Al Ramadhan S, Al Buali H, Al-Faraj J and \\ Al Mohanna M \\ Pediatric Department, Maternity Children Hospital, Saudi Arabia \\ *Address for Correspondence: \\ Al-buali MJ, Pediatric Consultant and Consultant of Medical \\ Genetics, Deputy Chairman of Medical Genetic Unite, Pediatrics \\ Department, Maternity Children Hospital, Al-hassa, Hofuf city, \\ Saudi Arabia; E-mail: doctormajed1@gmail.com \\ Submission: 15 July 2019 \\ Accepted: 5 August 2019 \\ Published: 9 August 2019 \\ Copyright: ๑ 2019 AL-Buali MJ, et al. This is an open access article \\ distributed under the Creative Commons Attribution License, which \\ permits unrestricted use, distribution, and reproduction in any medium, \\ provided the original work is properly cited.
}

of the most common groups of genetic diseases with a minimum prevalence of greater than 1 in 5000 in adults. Mitochondrial diseases can be present at birth but can be manifested also at any age [2] Whilst multi-system involvement is often evident, a neurological manifestation is the principal presentation in most cases. The multiple clinical phenotypes and the involvement of both the mitochondrial and nuclear genome make mitochondrial disease, particularly challenging for the clinician [3]. The clinical features are heterogeneous and often can mimic many neurological or other systemic diseases. The pediatric onset disease is associated with more severe multi-systemic involvement, relentless progression and poorer prognosis, however, there are rare exceptions, such as reversible respiratory chain deficiency caused by them. 14674T $>$ C mutation [4].

Most mitochondrial function and biogenesis are controlled by nuclear DNA. Human mitochondrial DNA encodes 13 proteins of the respiratory chain, while most of the estimated 1,500 proteins and components targeted to mitochondria are nuclear-encoded. Defects in nuclear-encoded mitochondrial genes are associated with hundreds of clinical disease phenotypes including anemia, dementia, hypertension, lymphoma, retinopathy, seizures, and neurodevelopmental disorders [5].

$\mathrm{NADH}$ dehydrogenase [ubiquinone] flavoprotein 1, mitochondrial (NDUFV1) is an enzyme that in humans is encoded by the NDUFV1 gene. The NDUFV1 gene encodes the $51-\mathrm{kD}$ subunit of complex I (NADH: ubiquinone oxidoreductase) of the mitochondrial respiratory chain. Defects in complex I are a common cause of mitochondrial dysfunction. Mitochondrial complex I deficiency is linked to myopathies, encephalomyopathies and neurodegenerative disorders such as Parkinson's disease and Leigh syndrome [6].

NDUFV1 is located on the $\mathrm{q}$ arm of chromosome 11 in position 13.2 and has 10 exons. The NDUFV1 gene produces a $50.8 \mathrm{kDa}$ protein composed of 464 amino acids [6,7].

Complex I am composed of 45 different subunits. NDUFV1 is a component of the Flavoprotein-Sulfur (FP) fragment of the enzyme. NDUFV1 is an oxidoreductase and a core subunit of complex I that is thought to be required for assembly and catalysis. It is a peripheral 
Citation: AL-Buali MJ, Al Ramadhan S, Al Buali H, Al-Faraj J, Al Mohanna M. Progressive Encephalopathy and Central Hypoventilation Related to Homozygosity of NDUFV1 Nuclear Gene, a Rare Mitochondrial Disease. J Pediatr Child Care. 2019;5(1): 05.

membrane protein located on the matrix side of the mitochondrion inner membrane [8]. Mutations in the NDUFV1 gene are associated with Mitochondrial Complex I deficiency, which is autosomal recessive. This deficiency is the most common enzymatic defect of the oxidative phosphorylation disorders $[9,10]$. Mitochondrial complex I deficiency shows extreme genetic heterogeneity and can be caused by a mutation in nuclear-encoded genes or in mitochondrial-encoded genes. There are no obvious genotype-phenotype correlations and inference of the underlying basis from the clinical or biochemical presentation is difficult, if not impossible. However, the majority of cases are caused by mutations in nuclear-encoded genes. It causes a wide range of clinical disorders, ranging from the lethal neonatal disease in adult-onset neurodegenerative disorders. Phenotypes include macrocephaly with progressive Leukodystrophy, nonspecific encephalopathy, hypertrophic cardiomyopathy, myopathy, liver disease, Leigh syndrome, Leber hereditary optic neuropathy, and some forms of Parkinson disease. Clinical manifestations can include lactic acidosis, cerebral degeneration, ophthalmoplegia, ataxia, spasticity, and distortion resulting from mutations in NDUFV1 [1114].

Here, we describe a clinical presentation of a patient with a rare autosomal recessive mitochondrial disease due to a homozygous mutation in the NDUFV1 gene, one of the nuclear encoded genes that code for mitochondrial components.

\section{Method}

\section{Human subject}

In the present study, A retrospective chart review was conducted as well, we clinically investigated affected individually (proband) from Saudi origin family. The proband underwent a comprehensive clinical evaluation by a general pediatrician, radiologist, neurologist and clinical geneticist.

\section{Molecular genetic test}

Analysis: More than 20.000 genes in the human genome were enriched using Roche/NlmbleGen technology (SeqCap MedExome Library) and sequenced on an alumina HISeq 1600 system (whole exome sequencing, WES) (details of the method at the end of the report). The aberration In the NDUFV1 gene was identified by filtering the exam data for homozygous variants, bioinformatically extracted HBO (homoz. ygosly-by-descent) regions and by a literature-based survey against the Indication of Interest.

Disturbances of mitochondrial energy metabolism occurs with an estimated incidence of 1 In 10,000 live births and are often caused by isolating mitochondrial complex I (NADH: ubiquinone oxidoreductase) deficiency, which causes a wide range of clinical disorders, ranging from the lethal neonatal disease in adult-onset Neuro degenerative disorders. Mitochondrial complex I deficiency shows extreme genetic heterogeneity. However, the genetic defects are thought to be mainly of nuclear origin, especially if the symptoms begin during Infancy. Mutations in the NDUFV1 gene are associated with Leukodystrophy and myoclonic epilepsy, which Is inherited In an autosomal-recessive manner.

Interpretation: WES revealed a homozygous $\mathrm{C}>\mathrm{T}$ transition at the position. 1268 in exon 9 of the NDUFV1 gene (c. 1268C>T). This missense mutation, which was confirmed by conventional Sanger sequencing, results in an amino acid exchange from threonine to methionine at position p. 423 (p. Thr423Met). An allele frequency in the general population has not been documented (ExAC), Allusedbio informatic programs (10/10) predict this alteration to be pathogenic. The mutation has already been described in two siblings, who carry the mutation in a compound heterozygous state with a nonsense mutation in exon 3 of the other allele (c. 175C >T; p. Arg59•). Both children presented at the age of five months with repeated vomiting and developed strabismus, progressive muscular hypotonia, myoclonic epilepsy and psychomotor regression. A cranial CT-Scan revealed brain atrophy. The boys died at 14 and 17 months from aspiration pneumonia (3). In summary, the homozygous missense mutation c.1268C $>\mathrm{T}$ (p.Thr423Met) In the NDUFV1 gene is probably responsible for the clinical phenotype of Aljubarah Abdulmalek.

In the case of parental consanguinity it is very likely that the mutation is indeed homozygous. To distinguish between homozygosity and hemizygoslty of the mutations. $1268 \mathrm{C}>\mathrm{T}$ (p.Thr423Met) with a large deletion on the other allele were commended analysis of both parents for the mutation. However, both scenarios are in accordance with the clinical phenotype of your patient. Targeted molecular genetic testing can be offered to further affected and unaffected family members of the patient.

Method: Genomic DNA was fragmented, and the exons of the known genes in the human genome as well as the corresponding exon-In iron boundaries were enriched using the Roche NimbleGen capture technology (SeqCap MedExome Libraiy), amplified and sequenced simultaneously by Ilumina technology (next-generation sequencing, NGS) using an Ilumina HiSeq 1600 system. The target regions were sequenced with an average coverage of 130-fold. For about $98 \%$ of the regions of interest 15 -fold coverage, for about $97 \%$ in 20-fold coverage was obtained. NGS data analysis was performed using Bioinformatics analysis tools as well as JSI Medical Systems software (version 4.1.2). Identified variants and ideal Indels were filtered against external and internal databases and filtered depending on their allele frequency, focusing on rare variants with a Minor Allele Frequency (MAF) of $1 \%$ or less. Nonsense, frameshift and canonical space site variants were primarily considered likely pathogenic. Assessment of pathogenicity of identifying non-synonymous variants were performed using bioinformatic prediction programs like mutation tester, Polyphen-2, Mutation Assessor, FATHMM etc. Only those variants were considered likely pathogenic which were predicted probably damaging by the majority of the used organisms. Variants that have been annotated as common polymorphisms in databases are in the literature were neglected.

Putatively pathogenic differences between the wild type sequence (human reference genome, according to the UCSC genome browser. hg19, GRCh37) and the patient's sequence mentioned and interpreted in this report were validated using Polymerase Chain Reaction (PCR). Amplification followed by conventional Sanger sequencing. The resulting sequence data for the NDUFV1 gene (OMIM 161015; locus; chromosome 11q13.2) was compared to the reference sequence NM_007103.3.

Restricted analysis: This initial analysis step was conducted with a homozygosity based strategy under the assumption of an autosomal 
Citation: AL-Buali MJ, Al Ramadhan S, Al Buali H, Al-Faraj J, Al Mohanna M. Progressive Encephalopathy and Central Hypoventilation Related to Homozygosity of NDUFV1 Nuclear Gene, a Rare Mitochondrial Disease. J Pediatr Child Care. 2019;5(1): 05.

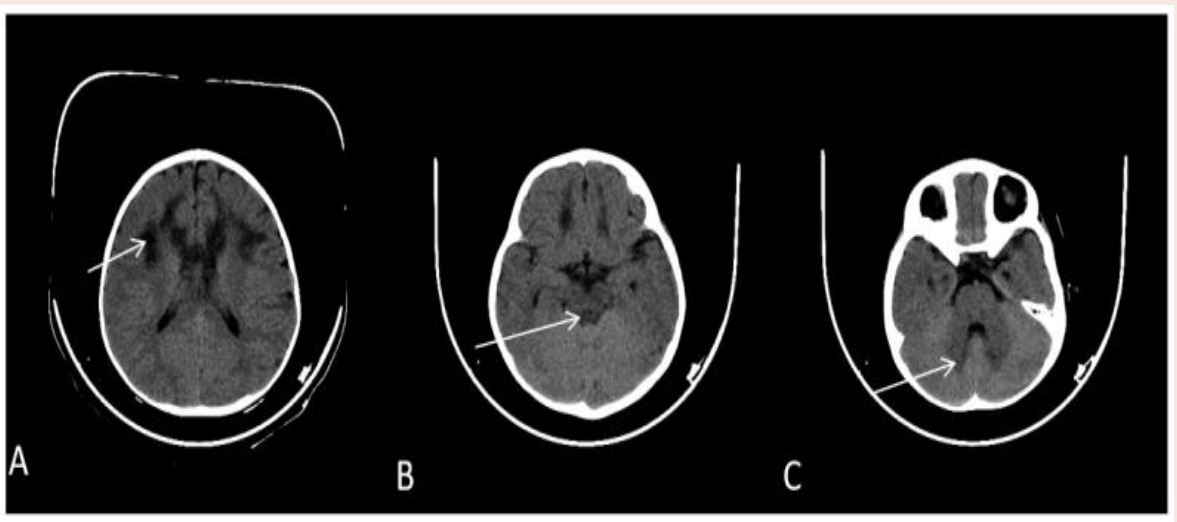

Figure 1: Axial plain CT scan of the brain show bilateral symmetrical hypo attenuating areas matching to the MRI high signal intensity at periventricular white matter $(A)$, periaqudect of the midbrain $(B)$, and dentate nucleus of the cerebllum $(C)$.

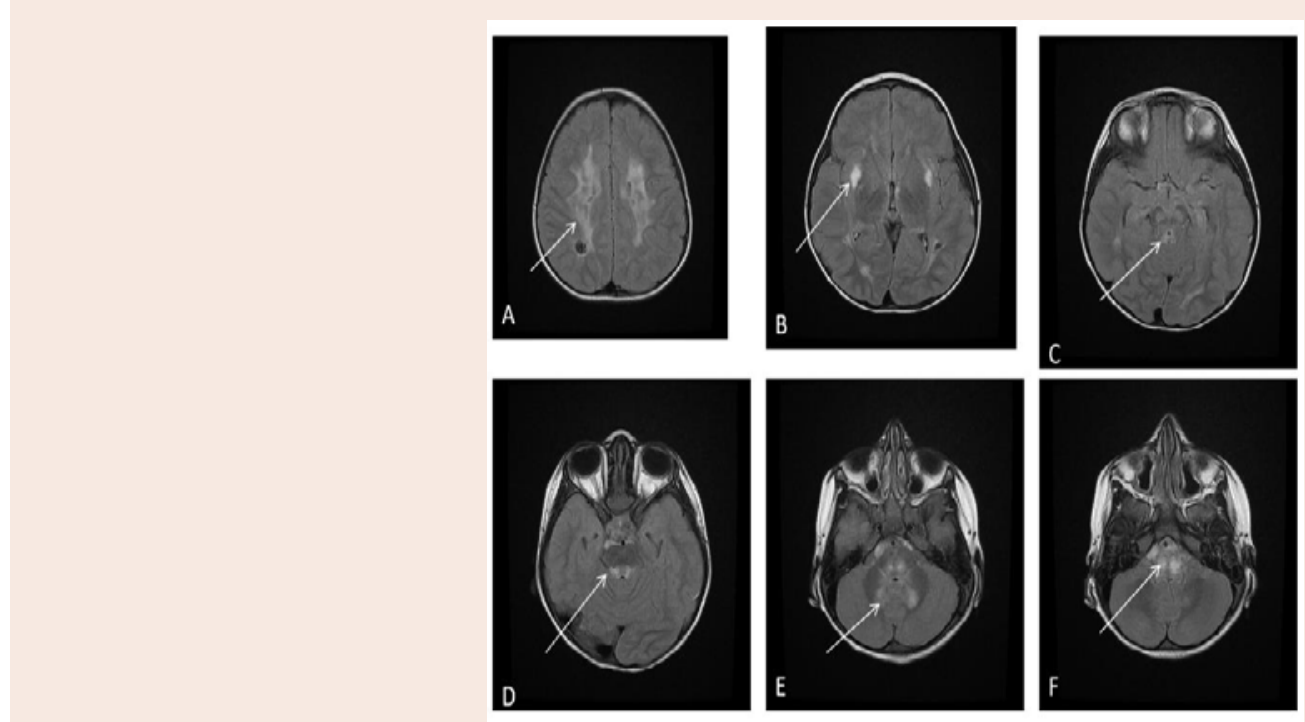

Figure 2: Axial MRI brain of different levels, T2 FLAIR pulse sequences that show bilateral symmetrical high signal intensity at periventricular white matter (A), putamen $(B)$, periaqueduct of midbrain $(C)$, posterior surface of the pons $(D)$, dentate nucleus of the cerebellum $(E)$, and medulla oblongata $(F)$.

recessive inheritance mode. Exam data were filtered against homozygous variants and HBD (homozygosity-by-descent) region extracted by Bioinformatics tools as large stretches of homozygous regions from informative SNPs in the data set. Furthermore, filtering against reported more allele frequencies in public databases while and the functional prediction score was conducted. Finally the condition in question is not evaluated. Incidental findings are not being reported routinely.

Limitations of WES: Whole exome sequencing is a rapidly evolving type of analysis. WES is being carried out using resources corresponding to the current technical and medical standards and scientific knowledge. Although the majority of the exam is sufficiently covered (about 90\%), Some regions remained poorly covered or maybe missed, And mutations in this region would escape detection. Coverage of WES data is partially for below target panel sequencing of genes for a distinct indication, mutations of the mitochondrialDNA and mutations in non-coding regulatory regions and deep intronic splice mutations can be missed. Due to missing specificity in the sequence capture approach coding regions for which highly homologous sequences exist in the genome are partially difficult to interpret, and Sanger sequencing of this region is not being conducted routinely.

The bioinformatic analysis still has some limitations regarding mapping, variant calling issues, detection of insertions/deletions or database infrastructure and is steadily improving. Currently applied analytical strategies might be hampered by failures from the limitations and assumptions of filtering variants as for example a large genetic heterogeneity of certain disorders, a reduced penetrance of certain mutations or a misinterpretation of variants may generate misleading results. Furthermore, too many candidate variants might remind after filtering without any functions or final proof been available at the limo of testing. Analysis and interpretation of WES data strongly depend on the availability of clinical data and family history. Clinical heterogeneity or incorrect diagnosis and family 

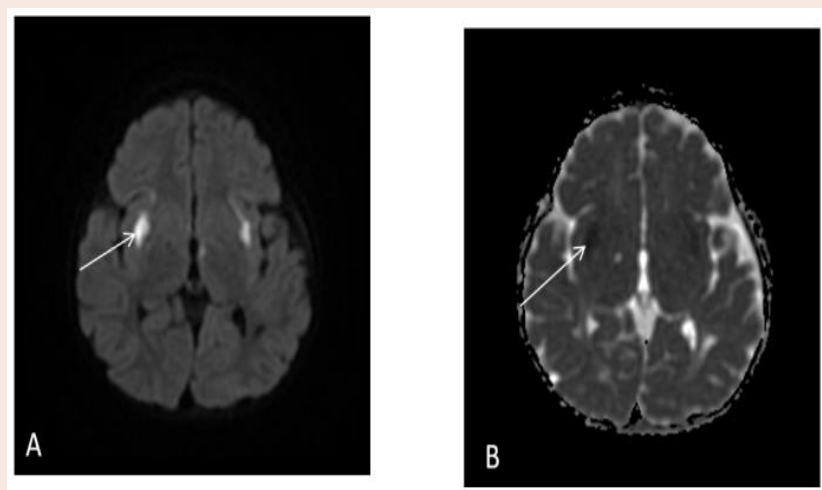

Figure 3: Active disease appear restriction as bilateral symmetrical putamen high signal intensity on Diffusion weighted image (A) and low signal intensity on Apparent diffusion co-efficient (B).

history may impact analysis strategies.

The knowledge about the causes or human genetic disorders constantly improves due to the continuous identification of novel disease genes. Repealed analysis or current exam date after a few years time with further developing analysis options and resources might lead to a more comprehensive and thereby differing result.

\section{Case Report}

Our present study, 36 months old girl presented with a history of failure of gain normal developmental milestones noticed at the age of 8 months. She was born full term via Cesarean section with birth weight $3 \mathrm{~kg}$ ( $3^{\text {rd }}$ centile). Her perinatal or postnatal history was unremarkable. Negative history for neonatal intensive care admission and she was discharged with mother in good condition. The patient was doing well, with normal development until age of 8 months; when she stopped gaining any developmental milestones. She was able to sit without support, but she was not able to crawl or stand. Gradually, she became hyperactive and had poor interaction over the next couple of months. Parents reported a history of abnormal movement in the form staring and loss of body tone for less than one minute, which was infrequent 2-3 times per month.

Later, the patient was admitted 2 times to Pediatric Intensive Care Unit (PICU) with a drop in the level of consciousness associated with central hypoventilation, suspicion of meningoencephalitis was raised so she was covered with antibiotics but all Cerebro-Spinal Fluid (CSF) studies and cultures were normal. Her first admission was at the age of 18 months, she kept ventilated for 3 months after the failure of frequent extubating trials. Then the patient was able to extubate herself accidentally as her level of consciousness improved. So, she was discharged with her family after the return of her baseline clinical condition.

By the time she was home, family stated (subjectively) that she started to show some improvement in her developmental milestones, she started to crawl and stand with support, she was able to say two word sentences. No history suggestive of feeding problems or chocking the girl evaluated by an ophthalmologist, which showed convergent squint with no retinal changes or nystagmus. The Hearing assessment was normal.

Her second admission was at the age of 36 months old, when she deteriorated again with encephalopathy and hyperventilation. She ventilated again, but weaning from the mechanical ventilator was unsuccessful, so she remained on a ventilator with chronic nursery care in the PICU.

Her parents are Second-degree cousins and this is their third child. She has a strong family history of mitochondrial disease. Her older sister died at the age of 14 months with a similar presentation of progressive encephalopathy before diagnosis was made. Her MRI finding shows white matter changes. (Any Central Hypoventilation?) The second sibling was having similar clinical presentation with white matter changes in the brain Magnetic Resonance Image (MRI), died at age 2 years (died of what? Any central hypoventilation?), WES was done for him and confirming mitochondrial disease due to a homozygous mutation of the nuclear gene NDUFV1 Parents (which variant-? Class?? If the patient underwent genetic testing and results showed both are heterozygous for the same mutation.

On examination at age 36 months, she was $16 \mathrm{~kg}$ ( $90^{\text {th }}$ centile), height $89 \mathrm{~cm}$ ( $25^{\text {th }}$ centile) and head circumference $46 \mathrm{~cm}$ at $\left(>5^{\text {th }}\right.$ centile). She is Normocephalic with no dysmorphism or neurocutaneous stigmata. She had a bilateral squint. She had generalized hypotonia, exaggerated deep tendon reflexes with positive ankle clonus bilaterally. There was no abnormal movement. She was connected to mechanical ventilators with low settings, fair air entry bilaterally and clear chest. Her liver was palpable $3 \mathrm{~cm}$ below costal margin liver span was around $7 \mathrm{~cm}$. Cardiovascular examination was unremarkable.

Basics work-up including complete blood count, renal and liver function tests were normal. Her metabolic workup including ammonia, lactate, Blood sugar and Tandem Mass Spectrometry (TMS) were normal. CSF analysis was normal. EEG was done at age 18 months and shows generalized slowing and no positive epileptiform activity. Brain CT showed symmetrical hypodensity involving the white matter of the frontal lobes, periventricular area and the genu of corpus callosum giving butterfly appearance and also noted focal hypodensity in the white matter of the left cerebellar hemisphere (Figure 1).

Magnetic Resonance Imaging of the brain (MRI) done at age 15 months with IV Gadolinium, shows bifrontal, Parietal, medial temporal, periventricular, genu of the corpus callosum, medial 
Citation: AL-Buali MJ, Al Ramadhan S, Al Buali H, Al-Faraj J, Al Mohanna M. Progressive Encephalopathy and Central Hypoventilation Related to Homozygosity of NDUFV1 Nuclear Gene, a Rare Mitochondrial Disease. J Pediatr Child Care. 2019;5(1): 05.

thalami, midbrain, dorsal pons, dentate Nuclei and Periaueductal, superior cerebellar peduncle abnormal high signal intensity with restricted diffusion, and some them shows cystic changes. No abnormal enhancement identified. No hydrocephalus was found (Figures 2 and 3).

Finally, to reach a definitive diagnosis and to rule out other possibilities of presenting phenotype and neuroradiological changes, molecular genetics study has been done through (WES) which showed Missense mutation c. $1268 \mathrm{C}>\mathrm{T}$ (P. The $423 \mathrm{Met}$ ) in axon 9 of the NDUFV1 gene in the homozygous state, which is classified as causative for the patient phenotypes?

\section{Discussion}

The current patient is the first case to be reported from Saudi Arabia. The broadband has the clinical presentation suggesting of mitochondrial infancy Neuro-regression, unexplained encephalopathy, as well as seizure disorder. Neurological manifestations are the main phenotype features; Central hypoventilation is the main presenting morbidity. Central hypoventilation not explained by any other systemic disorder, it was frequent and progressive with time necessitated keeping the patient on mechanical ventilation.

These presentations can be manifested by many neurological diseases in pediatrics caused by genetic cause or as a squeal of acute acquired brain insult as perinatal asphyxia as well as meningoencephalitis. However, the consanguinity of the parent and family history of similar presentation with early childhood deaths are highly suggestive of inherited genetic disease.

The radiological finding in the present study showed Brain CT showed symmetrical hypodensity involving white matter, these mentioned findings consisted of metachromatic Leukodystrophy, biotin responsive basal ganglia encephalopathy, Leigh disease differential diagnosis.

At this point of work-up, the advanced molecular cytogenetic test is indicted. MtDNA the MtDNA gene test panel rated no significant variants. Whole Exome Sequence has been done in the present study, which showed the homozygous mutation in the nuclear encoded gene NDUFV1 c. $1268 \mathrm{C}>\mathrm{T}$ p. (Thr423Met) in the NDUFV1 gene (OMIM: 618225 ) in the proband and showed same mutation in the parent in heterozygous pattern. From our mini review, a few cases about NDUFV1 gene related encephalopathy have been reported in the literature. Compound heterozygous described mutations were more described $[13,14]$. Finally, WES it very useful to reach a diagnosis as well to find an explanation to the family for the presence of more than one sibling with this neurodevelopmental disease was given the couple a chance for Preimplantation Genetic Diagnosis (PGD).

\section{Conclusion}

We suggest that the clinicians should consider the possibility of mitochondrial diseases in the patient presented with unexplained progressive encephalopathy and hyperventilation. Molecular genetics test specifically Whole Exam Sequence (WES) is very helpful to reach a definitive diagnosis and to rule other differential diagnosis. Furthermore to delineate the long term medical care, the outcome and genetic counseling.

Finally, we emphasize the need for longitudinal data, as such information will provide a profile encompassing care recommendation, Future research is needed in order to elucidate the long-term outcome of these patients.

\section{References}

1. Dimauro S, Davidzon G (2005) Mitochondrial DNA and disease. Ann Med 37: 222-232.

2. Blau N, Duran M, Gibson KM, Dionisi-Vici C (2014) Physician's Guide to the Diagnosis, Treatment, and Follow-Up of Inherited Metabolic Diseases. Springer pp: 339.

3. Ng YS, Turnbull DM (2016) Mitochondrial disease: genetics and management. J Neurol 263: 179-191.

4. Horvath R, Kemp JP, Tuppen HA, Hudson G, Oldfors A, et al. (2009) Molecular basis of infantile reversible cytochrome c oxidase deficiency myopathy. Brain 132: 3165-3174.

5. Scharfe C, Lu HS, Neuenburg JK, Allen EA, Li GC, et al. (2009) Mapping gene associations in human mitochondria using clinical disease phenotypes. PLoS Comput Biol 5: e1000374.

6. Spencer SR, Taylor JB, Cowell IG, Xia CL, Pemble SE, et al. (1992) The human mitochondrial $\mathrm{NADH}$ : ubiquinone oxidoreductase $51-\mathrm{kDa}$ subunit maps adjacent to the glutathione S-transferase P1-1 gene on chromosome 11q13. Genomics 14: 1116-1118.

7. Zafeiriou DI, Rodenburg RJ, Scheffer H, van den Heuvel LP, Pouwels PJ, et al. (2008) MR spectroscopy and serial magnetic resonance imaging in a patient with mitochondrial cystic leukoencephalopathy due to complex I deficiency and NDUFV1 mutations and mild clinical course. Neuropediatrics 39: $172-175$.

8. Murray J, Zhang B, Taylor SW, Oglesbee D, Fahy E, et al. (2003) The subunit composition of the human NADH dehydrogenase obtained by rapid one-step immunopurification. J Biol Chem 278: 13619-13622.

9. McFarland R, Kirby DM, Fowler KJ, Ohtake A, Ryan MT, et al. (2004). De novo mutations in the mitochondrial ND3 gene as a cause of infantile mitochondrial encephalopathy and complex I deficiency. Ann Neurol 55: 58-64.

10. Schuelke M, Smeitink J, Mariman E, Loeffen J, Plecko B, et al. (1999) Mutant NDUFV1 subunit of mitochondrial complex I causes leukodystrophy and myoclonic epilepsy. Nat Genet 21: 260-261.

11. Kirby DM, Salemi R, Sugiana C, Ohtake A, Parry L, et al. (2004) NDUFS6 mutations are a novel cause of lethal neonatal mitochondrial complex I deficiency. J Clin Invest 114: 837-845.

12. de Coo RF, Buddiger P, Smeets HJ, van Oost BA (1997) Molecular cloning and characterization of the human mitochondrial NADH: oxidoreductase 10kDa gene (NDUFV3). Genomics 45: 434-437.
Acknowledgement

The authors would like to acknowledge the treating team as well as the parent of the patient for their kind cooperation. 